\title{
Scar Pregnancy- A Tertiary Care Experience and Review of Literature
}

\section{Lajya Devi Goyal}

\begin{abstract}
Incidence of Cesarean scar pregnancy is on rise due to increasing incidence of caesarean section. It is rare type of ectopic pregnancy associated with severe complications such as sever hemorrhage, uterine rupture, loss of fertility.Therapeutic options include, medical management with methotrexate, Ultrasuond guided injection of potassium chloride, Dilatation \& Curetage, laparoscopy laparotomyf and more recently uterine artery embolization. This study describes three case s of scar pregnancy presented at different gestational age and managed by different methods, one conservative medical method with injection methotrexate systemically, second with local resection of mass and repair in emergency and third with suction evacuation in emergency.
\end{abstract}

Keywords: Scar pregnancy, Ultrasonograhpy, $h C G$

\section{INTRODUCTION}

Cesarean scar pregnancy (CSP) is defined as implantation of gestational sac into the myometrium and the fibrous tissue of a previous cesarean delivery. With increasing incidence of caesarean sections, number of scar pregnancies is on the rise. We encountered 3 cases of CSP among 3256 deliveries making an incidence of 1 in 1085 deliveries and 1 in 218 among the women with previous scar. Seow et al estimated that the incidence of CSP was 1 per 2216 pregnancies and $0.15 \%$ for women with a previous CS[1] . Jurkovic et al have estimated the prevalence of CSP in local population attending the early pregnancy $1: 1800[2]$. As CSP can lead to catastrophic complications, early diagnosis and treatment is very important to reduce life-threatening complications. However, till date there is no consensus on the preferred therapeutic protocol. The management options includes medical methods like systemic or local administration of methotrexate (MTX), injection of Kcl into sac/intracardiac and surgical methods like uterine suction curettage, local resection of the mass, hysterectomy, and interventional radiology as uterine artery embolization (UAE) [3-5].

We report a series of three case s of scar pregnancy presented at different gestational age and managed by different methods, one conservative medical method with injection methotrexate systemically, second with local resection of mass and repair in emergency and third with suction evacuation in emergency.

\section{CASE -1}

38year $\mathrm{G}_{3} \mathrm{P}_{2} \mathrm{~L}_{2}$ reported to outpatient department of our hospital at 32days of gestation for medical termination of pregnancy. Obstetric history revealed previous two cesarean section and last child birth was eight years back. . Her menstrual cycle were regular 24days frequency. Pregnancy test was positive two days following missed period. Per vaginum finding revealed normal sized uterus and bilateral fornices were free. Ulrasonography reported gestational sac of 4wks 4dayswith at scar site. Serum $\beta$ hCG was ordered along with complete blood count, urine examination, renal function test and liver function test. Levels Serum $\beta$ hCG-3200IU/l , blood count RFT,LFT and urine test were in normal range. Patient was planned for medical management with methotrexate. Injection methotrexate $(65 \mathrm{mg})$ was injected. Patient developed mild mucositosis on fourth day . After one week repeat USG showed persistent sac, but no fetal cardiac activity appeared .Serum $\beta$ hCG levels were3000IU/1 and injection methotrexate was repeated in same dose. Serial $\beta$ hCG monitoring showed slow falling levels but higher levels in the range of $2400 \mathrm{iu} / 1,1800 \mathrm{iu} / 1$ in first and $2^{\text {nd }}$ week after the second dose respectively and persistent sac with no fetal cardiac activity. Patient refused further methotrexate therapy and evacuation and opted for serial Hcg monitoring. After 6weeks of second dose of methotrexate $\beta$ hcg levels were $600 \mathrm{iu} / \mathrm{l}$. She expelled the sac during first menstrual period 6 weeks after the therapy. Two week following menstruation her $\beta$ Hcg became negative. 


\section{CASE 2}

24 year G2P1L1 presented in our hospital with USG report of scar pregnancy at 10weeks \pm 3 days of gestation. She had previous LSCS one year back due to non progress of labour. She resumed menstruation 3 months after LSCS and had regular cycles. USG was repeated in our institute which revealed 11 weeks gestational sac involving scar site. Per vaginum(P/V) examination was not done keeping in mind the possibility of disturbance of pregnancy with cervical excitation. Patient was admitted and investigated. CBC revealed $\mathrm{Hb} 6 \mathrm{gm} \%$, TLC and platelet count was normal peripheral blood film revealed hypochromic microcytic anemia.. Renal function test, liver function test and urine routine examination were in normal range. $\beta$ Hcg levels were 11000iu/l. Patient was transfused with 4 units of packed RBCS. Hb was normalised and patient was planned for USG guided fetal Intracardiac potassium chloride injection. As we attempted to focus fetal heart by transabdominal probe and introduced needle into the sac patient developed severe bradicardia. She had profuse perspiration and started bleeding per vaginum. Patient was resuscitated and taken for emergency surgical therapy. In operation room under anaesthesia $\mathrm{p} / \mathrm{v}$ finding revealed bulge in the upper part of cervix and profuse bleeding. Laparotomy was done. Intraoprative finding showed placental tissue bulging at scar site and papery thin scar, scar was excised and product of conception were evacuated. Placenta was adherent at scar site was removed piece meals and bleeding was controlled with packing. Pack was removed later on and patient had uneventful recovery.

\section{CASE 3}

26years G3 P2 L1 was referred to our hospital with USG report of scar pregnancy at 6 weeks 6 days of gestation with left ovarian dermoid cyst. Patient had taken tab mifegest $200 \mathrm{mg} 24$ hour prior to the admission to our institution on advice of a private practioner. Her first delivery was normal vaginal delivery three years back having female healthy child. In second pregnancy 6 months back she had antepartum haemorrhage at $30 \mathrm{wks}$ gestation and underwent LSCS for major degree placenta praevia. A large right sided ovarian cyst was noted during caesarean section and right salpingopherectomy was done. Left ovary was normal looking. Histopathology was not available but patient was told benign nature of the ovarian mass. Neonate died due to prematurity. The present pregnancy she conceived within six months of caesarean section. USG was repeated in our institute and revealed same findings. CBC, urine routine RFT, LFT were in normal range. Serum $\beta$ Hcg 5467iu/L. We planned USG guided fetal intracardiac Kcl injectionfollowing morning, but patient started bleeding profusely. She was taken up in emergency operation theatre and evacuation was done. Check curettage was done and with injection ergometrine and prostadin bleeding was controlled and patient was revived. Post operative USG revealed no retained products. Patient was discharged next day in satisfactory condition.

\section{DisCUSSION}

CSP is associated with a high risk of serious complications, Due to rising trends of ceserean section and increased utilisation of ultrasound in modern era incidence of CSP is likely to rise exponentially. The fate of scar pregnancy may lead to catastrophic hemorrhage in early pregnancy and if it continues to grow may end into a low-lying adherent placenta with or without invasion of surrounding organs [6]. Early diagnosis is thus important to avoid serious complications. The sonographic criteria for early diagnosis of cesarean scar pregnancies in the first trimester include, an empty uterus with a clearly visualized endometrium;, empty cervical canal;gestational sac within the anterior portion of the lower uterine segment at the presumed site of the cesarean scar; and thinned or absent myometrium between the gestational sac and bladder ( $<5 \mathrm{~mm}$ in two-thirds of cases)[7]

The best management option is still unclear and individualised depending on gestational age and presentation. The main objectives in the management should be the prevention of massive blood loss and the conservation of reproductive potential. Recent data emphasize on early diagnosis and suggest single or combined medical and surgical treatment options preventing uterine rupture and haemorrhage and preserving the fertility. Medical management includes administration of drugs such as KCL, MTX, mifepristone and monitoring of the serum $\beta$-HCG levels. Drawbacks of medical management includes firstly, to normalise serum level of $\beta$-HCG it takes 2 months to 6 months.Second Vaginal bleeding and gestational sac may persist for 2-3 months, third the failure rate in medical treatment is very high and surgery may be required in such cases.[5,7]. Our first case was managed medically and similar problems were encountered. Surgical techniques may be warranted in 
emergency situations where the patient reports in state of shock due to profuse bleeding as in second in third case of our series. Depending upon the clinical scenario patient may require dilation and curettage, laparotomy, and hysterectomy. But due to increased risk of complications such as massive hemorrhage, uterine perforation, shock, and even death these methods are not chosen as elective procedures[8-10]. Minimal invasive surergy such as laparoscopic CS gestational product resection, hysteroscopic removal gestational sac have high failure rate and morbidity therefore not preferred mode of management[11] . Newer surgical method of surgical management described by Zhang et al who successfully treated 40 cases of CSP with transvaginal hysterotomy without significant complication [12].

Uterine artery embolisation (UAE) has been widely used for the treatment of postpartum haemorrhage, cervical pregnancy, leiomyoma [13-15]. Recent literature suggest use of UAE as effective method of treatment of CSP $[5,15]$. Zhuang Y et al compared the uterine

artery embolization with methotrexate for the management of pregnancy and reported less blood loss with UAE followed by suction curettage compared to methotrexate[16]. Yan Huang et al. described chemoembolisation through uterine arteries followed by suction evacuation after 24-48 hours as effective method of management of CSP[17]. Drawbacks of UAE includes, first of all it is not widely available in all institutes of resource limited countries like India, and secondly it is not a risk free procedure. Apart from mild complication as pain, nausea vomiting, ovarian perfusion is the real concern about the reproductive potential of the patient [15].

\section{SumMary}

Early diagnosis of caesarean scar pregnancy and its effective management is important to preserve the fertility of patients and reduce maternal mortality. There are no universal treatment guidelines for the management of CSP till now. Every mode of treatment has its own risk and benefits and treatment is individualised. Further large and multicentre studies are required for establishment of recommendations.

\section{REFERENCES}

[1] Seow KM, Huang LW, Lin YH, Lin MY, Tsai YL, Hwang JL. Cesarean scar pregnancy: issues in management. Ultrasound Obstet Gynecol. 2004;23:247-25

[2] Jurkovic D, Hillaby K, Woelfer B, et al. First-trimester diagnosis and management of pregnancies implanted into the lower uterine segment Cesarean section scar. Ultrasound Obstet Gynecol. 2003; 21:220-227.

[3] Litwicka K, Greco E. Caesarean scar pregnancy: a review of management options. Curr Opin Obstet Gynecol. 2013; 25:456-461.

[4] Kai K, Shimamoto K, Matsumoto H, Narahara $\mathrm{H}$. Conservative surgical treatment for caesarean scar pregnancy. J Obstet Gynaecol. 2014; 34:91-92.

[5] Singhal S, Singh A, Raghunandan C, Gupta U, Dutt S. Uterine artery embolization: exploring new dimensions in obstetric emergencies. Oman Med J. 2014;29:217-219

[6] A.Al-Nazer, L. Omar, M. Wahba, T. Abbas, and M. Abdulkarim, "Ectopic intramural pregnancy developing at the site of a cesarean section scar: a case report," Cases Journal, $2009 ; 2 ; 12$, 9404,

[7] Ash A, Smith A, Maxwell D. Caesarean scar pregnancy. BJOG 2007; 114:253-263

[8] Shufaro Y, Nadjari M. Implantation of a gestational sac in a cesarean section scar. Fertil Steril.2001; 75:1217.

[9] Rotas MA, Haberman S, Levgur M. Cesarean scar ectopic pregnancies:etiology, diagnosis, and management. Obstet Gynecol. 2006; 107:1373-1381.

[10] Arslan M, Pata O, Dilek TU, Aktas A, Aban M, Dilek S. Treatment of viable cesarean scar ectopic pregnancy with suction curettage. Int J Gynaecol Obstet. 2005;89:163-166.

[11] Wang CJ, Chao AS, Yuen LT, Wang CW, Soong YK, Lee CL. Endoscopic management of cesarean scar pregnancy. Fertil Steril. 2006;85:494,

[12] Zhang Huanxiao, Chen Shuqin, Jiang Hongye, Xie Hongzhe, Niu Gang, Xu Chengkang, Guan Xiaoming, and Yao Shuzhong Transvaginal hysterotomy for cesarean scar pregnancy in 40 consecutive cases. Gynecol Surg. 2015; 12(1): 45-51. 
[13] Ravina JH, Herbreteau D, Ciraru-Vigneron N, Bouret JM, Houdart E, Aymard A, Merland JJ. Arterial embolisation to treat uterine yomata. Lancet. 1995;346:671- 672

[14] Pelage JP, Le Dref O, Jacob D, Soyer P, Herbreteau D, Rymer R. Selective arterial embolization of the uterine arteries in the management of intractable post-partum hemorrhage. Acta Obstet Gynecol Scand.1999; 78:698-703.

[15] Xiaolin Z, Ling L, Chengxin Y, Yiqing T, Jun W, Yan C, Guangxi T. Transcatheter intraarterial methotrexate infusion combined with selective uterine artery embolization as a treatment option for cervical pregnancy. J Vasc Interv Radiol. 2010;21:836-841

[16] Zhuang Y, Huang L. Uterine artery embolization compared with methotrexate for the management of pregnancy implanted within a cesarean scar. Am J Obstet Gynecol. 2009; 201:152.e1-3.

[17] Yan Huang, Youfei Li, Rongkai Xi, Zhengqiong Chen, Demei Ying, Zhen Li, and Ying Yang. An application of uterine artery chemoembolization in treating cesarean scar pregnancy. Int $\mathbf{J}$ Clin Exp Med. 2015; 8(2): 2570-2577 